\title{
Pattern Formation on Silicon-on-Insulator
}

Frank S. Flack ${ }^{1}$, Bin Yang ${ }^{1}$, Minghuang Huang ${ }^{2}$, Matt Marcus ${ }^{1}$, Jason Simmons ${ }^{1}$, Olivia M. Castellini ${ }^{1}$, Mark A. Eriksson ${ }^{1}$, Feng Liu ${ }^{2}$, Max G. Lagally ${ }^{1}$

${ }^{1}$ Materials Research Science and Engineering Center

University of Wisconsin

Madison, WI 53706, U. S. A.

${ }^{2}$ University of Utah

SaltLake City, UT 84112, U. S. A.

\section{ABSTRACT}

The strain driven self-assembly of faceted Ge nanocrystals during epitaxy on $\mathrm{Si}(001)$ to form quantum dots (QDs) is by now well known. We have also recently provided an understanding of the thermodynamic driving force for directed assembly of QDs on bulk Si (extendable to other QD systems) based on local chemical potential and curvature of the surface. Silicon-on-insulator (SOI) produces unique new phenomena. The essential thermodynamic instability of the very thin crystalline layer (called the template layer) resting on an oxide can cause this layer, under appropriate conditions, to dewet, agglomerate, and self-organize into an array of Si nanocrystals. Using low-energy electron microscopy (LEEM), we observe this process and, with the help of first-principles total-energy calculations, we provide a quantitative understanding of this pattern formation. The Si nanocrystal pattern formation can be controlled by lithographic patterning of the SOI prior to the dewetting process. The resulting patterns of electrically isolated Si nanocrystals can in turn be used as a template for growth of nanostructures, such as carbon nanotubes (CNTs). Finally we show that this growth may be controlled by the flow dynamics of the feed gas across the substrate.

\section{INTRODUCTION}

Silicon-on-insulator substrates are formed by a $\mathrm{SiO}_{2}$ layer sandwiched between a thin crystalline top Si layer (the template layer) and a thick Si (handle) wafer. This arrangement makes it very attractive for use as a substrate in the fabrication of low-power, low-voltage devices and advanced devices requiring thin geometries. As devices shrink in lateral size, the template layer must shrink correspondingly in order to maintain the benefits of an SOI structure. This ultra-thin configuration, however, is only quasi-stable, thermodynamically. As the template film is made thinner, the surface free energy begins to overcome the kinetic limitations on its geometry [1-4]. Upon heating, such films may act to minimize their surface free energy by changing their morphology, typically via dewetting the unstable interface and agglomeration into 3D nanocrystals (see review [5]). Such behavior has obvious implications for semiconductor device processing, but can potentially be exploited to generate useful nanostructures as well.

Earlier work on SOI decomposition has noted that template layers on the order of tens of nanometers in thickness will agglomerate in patterns that are aligned in the $<310>$ directions, but has not been able to explain this behavior. This study proposes a mechanism describing the 
source of ordering of nanocrystals into networks by thermal dewetting of SOI and presents a novel application for such structures as templates for ordered growth of carbon nanotubes.

\section{EXPERIMENTAL DETAILS}

Silicon-on-insulator wafers are commercially available with template layer thicknesses on the order of hundreds of nanometers. However, because we are investigating processes occurring on reduced scales, we must first thin the template layers by dry thermal oxidation followed by wet chemical etching. The final substrates consist of a thick Si handle wafer covered with $200 \mathrm{~nm}$ $\mathrm{SiO}_{2}$ and a single-crystal template layer tens of nanometers thick. All SOI wafers used in this study were wafer-bonded with a (001) orientation.

The wafers are chemically cleaned using standard procedures, [6] introduced into the LEEM chamber at a base pressure of $5 \times 10^{-10}$ Torr and outgassed overnight at $650^{\circ} \mathrm{C}$. The cleaning procedure covers the sample with a thin oxide layer which protects the Si template layer from decomposition at these lower temperatures. The sample is rapidly heated to $900^{\circ} \mathrm{C}$ and held for 50 seconds in a disilane atmosphere at $5 \times 10^{-8}$ Torr to remove the oxide, then cooled to $650^{\circ} \mathrm{C}$ to be imaged by LEEM. We varied the temperature between $650^{\circ} \mathrm{C}$ and $980^{\circ} \mathrm{C}$ to change the thermal decomposition rate of the Si template layer, while monitoring the sample with LEEM in dark-field imaging mode. In this mode, contrast arises from the electron emission difference between $\mathrm{Si}$ and $\mathrm{SiO}_{2}$ [7]. Therefore, the $\mathrm{Si}$ template surface appears dark and the exposed oxide surface appears bright.

Nanotubes are grown on decomposed SOI substrates by chemical vapor deposition (CVD) at a substrate temperature of $900^{\circ} \mathrm{C}$ with flows of $\mathrm{CH}_{4}$ at $400 \mathrm{sccm}$ and $\mathrm{H}_{2}$ at $20 \mathrm{sccm}$ near atmospheric pressure. Either a Fe-Pt salt solution (following the recipe in [8], diluted $10^{6}$ times) is deposited or a thin Fe film is evaporated on this substrate to act as the catalyst for CNT growth. Samples were then imaged by scanning electron microscopy (SEM) or atomic force microscopy (AFM).

\section{DISCUSSION}

Observations of decomposition and agglomeration of amorphous or polycrystalline silicon films have shown that the resulting nanocrystal distribution tends to have little or no organization [9-12]. Though such distributions may have a preferred growth direction, the initial polycrystalline structure induces an overall randomness in lateral structure. Single-crystal films, however, are typically grown epitaxially in a layer-by-layer mode (Frank-van der Merwe growth), indicating the strong attraction between the substrate and epilayer.

SOI is single-crystal but not epitaxial, therefore the film can decompose without the randomness inherent in polycrystalline and amorphous films. This supplies a source of order to the distribution of agglomerated islands stemming solely from the energetics of the Si crystal. As the Si template layer thickness in SOI is reduced to the order of tens of nanometers, its thermal stability decreases. At elevated temperatures in high vacuum, the thin Si template layer dewets and beads up into Si nanocrystals, which self-order into a very distinctive pattern on the underlying amorphous oxide substrate as seen in figure 1. Although this pattern formation has been observed before $[13,14]$, the mechanism has not been explained. 

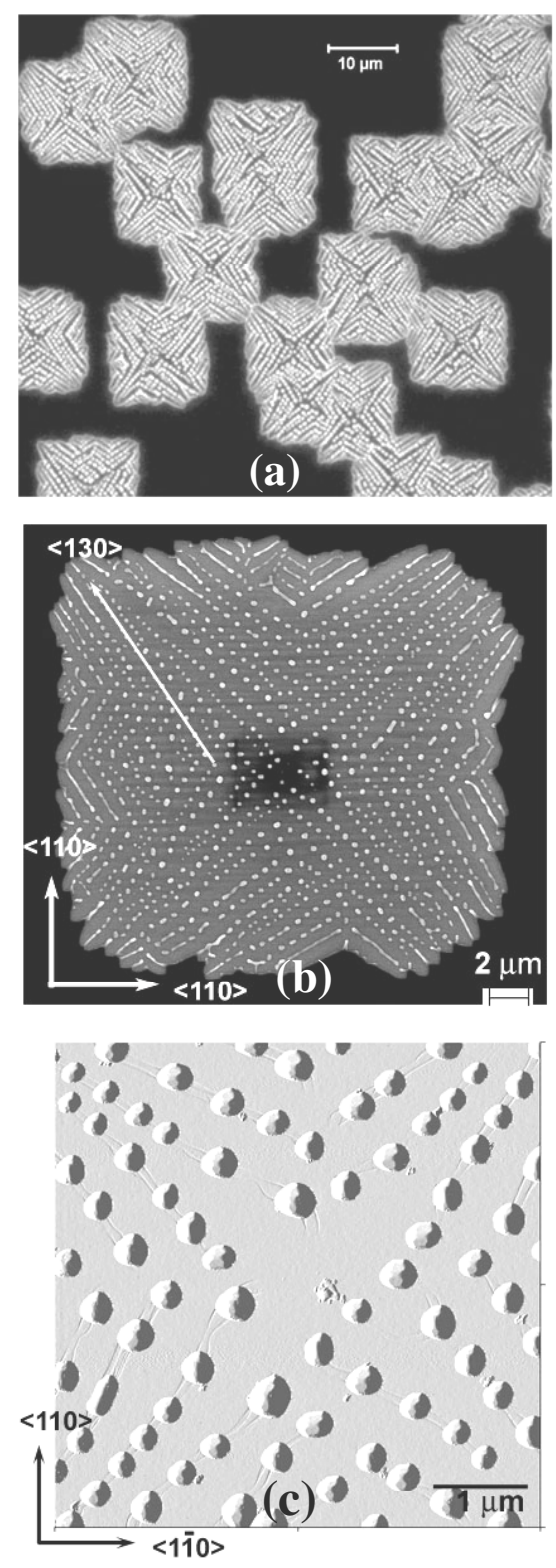

Figure 1. Self-organized patterns of Si nanocrystals from agglomeration of a $9 \mathrm{~nm}$ thick SOI. (a) Optical image showing patterns of an agglomerated SOI wafer (1000x). (b) SEM image showing an ordered array of Si nanocrystals (bright spots) in one agglomerated pattern. (c) AFM image showing the center region of one pattern. The Si nanocrystals are faceted and $90 \mathrm{~nm}$ high and $300 \mathrm{~nm}$ wide on the average.

\section{Formation of nanocrystal networks by thermal dewetting of SOI}

Dewetting of a thin film results from thermal diffusion of surface atoms from the surface facet to more energetically favorable ones. Some disagreement exists as to which facets exhibit the lowest free energy in silicon. Early predictions showed that the $\{111\}$ facets were the lowest in energy but, more recently, the $\{311\}$ facets have been found to be comparable, depending upon the temperature $[15,16]$. These surface energies have been calculated from first principles,. 
but only separately by different groups [17-19]. Comparison between them is not reliable because the energies calculated for these surfaces differ by only a few meV, which is within the uncertainty of a given first-principles calculation. To make a direct comparison, we have calculated the surface energies systematically using the same method [17] and equivalent parameter settings to minimize the errors. Our calculations predict that the $\mathrm{Si}(111)$ and $\mathrm{Si}(311)$ surfaces are almost degenerate at lower temperatures; but that both the $\mathrm{Si}(311)-(3 \times 1)$ and $\mathrm{Si}(311)-(1 \mathrm{x} 1)$ reconstructions are more stable than the $\mathrm{Si}(111)$ surface for the higher temperatures at which the thermal dewetting takes place [20].

The selective faceting process yielding a predominant (311) facet is confirmed by AFM measurement of surface contact angles of the Si islands, as shown in figure 2. AFM line scans show a surface contact angle of $72 \pm 2^{\circ}$, indicating the large facets at the island base are indeed (311) facets. As seen in figure 1a, the islands are multifaceted. The shallower (111) and (113)
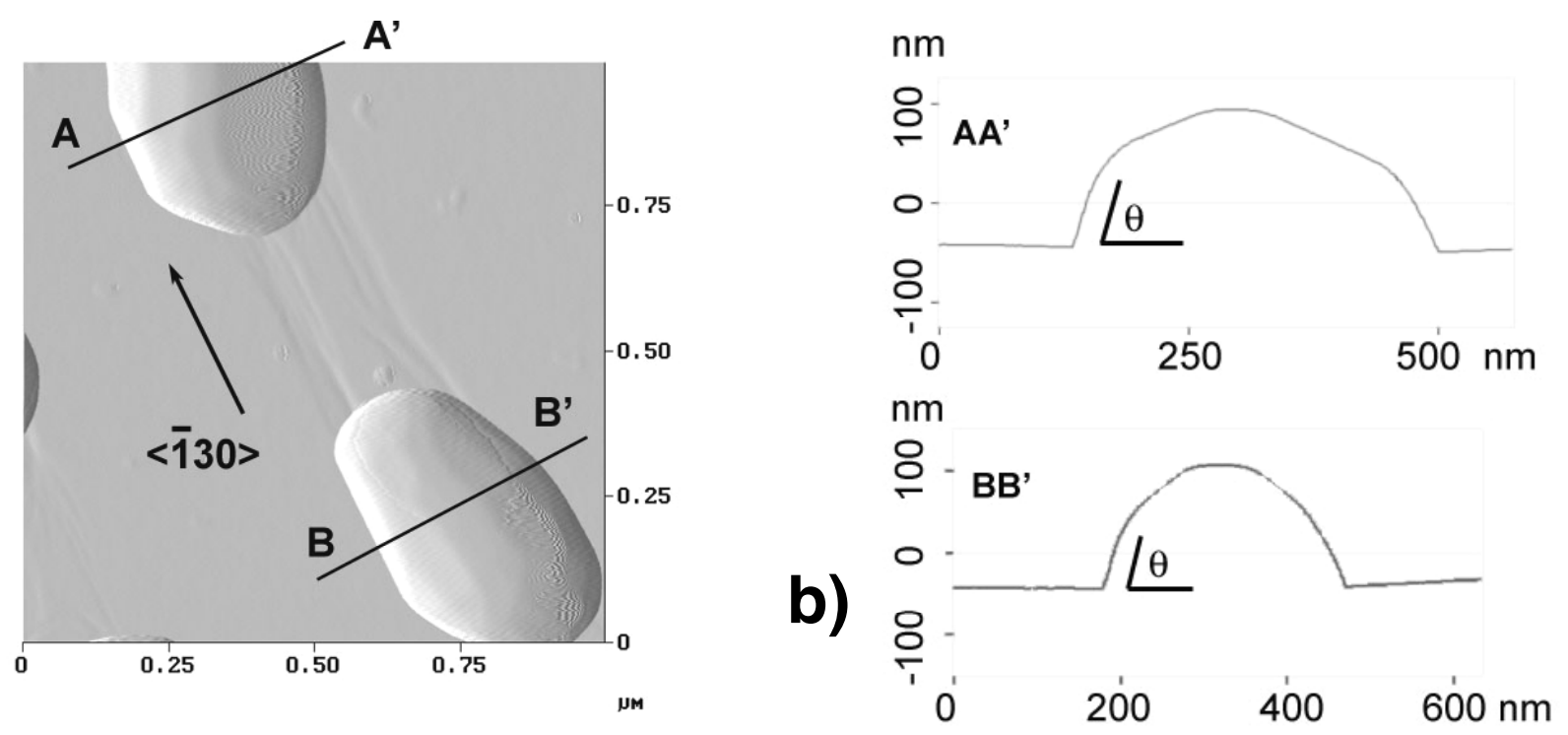

Figure 2. a) An AFM image of a typical silicon nanocrystal formed by SOI decomposition at $980^{\circ} \mathrm{C}$ and, b) its cross section. The $72^{\circ}$ angle with the (001) substrate corresponds to a (311) facet. The facet on the right side of the island appears shallower due to the asymmetry of the AFM tip.

facets on the top of the islands are formed as the low-energy facet nearest in angle $\left(\right.$ at $25.24^{\circ}$ ) to the (001) surface plane. Because the energy of both (311) and (113) facets are the same, the formation of each is an indication of the starting facet. The higher-angle (311) facet stabilizes first because the sidewalls formed from an initial near-vertical trench. The top of the islands is formed from atoms diffusing from the (001) surface and so the shallower (113) facet is formed.

Figure 3 shows four real-time LEEM images of a dewetting boundary front at five-second intervals with a substrate temperature of $980^{\circ} \mathrm{C}$. The dark regions are the silicon template layer and nanocrystals and the light areas are the exposed $\mathrm{SiO}_{2}$. The $\mathrm{Si}$ layer dewets the oxide in stripes along the [130] directions, beginning at the edges of the complete Si layer, with Si atoms diffusing away to leave exposed oxide trenches. Because the channels are directed along the [130] directions, the sidewalls must have $\{311\}$ facets. 


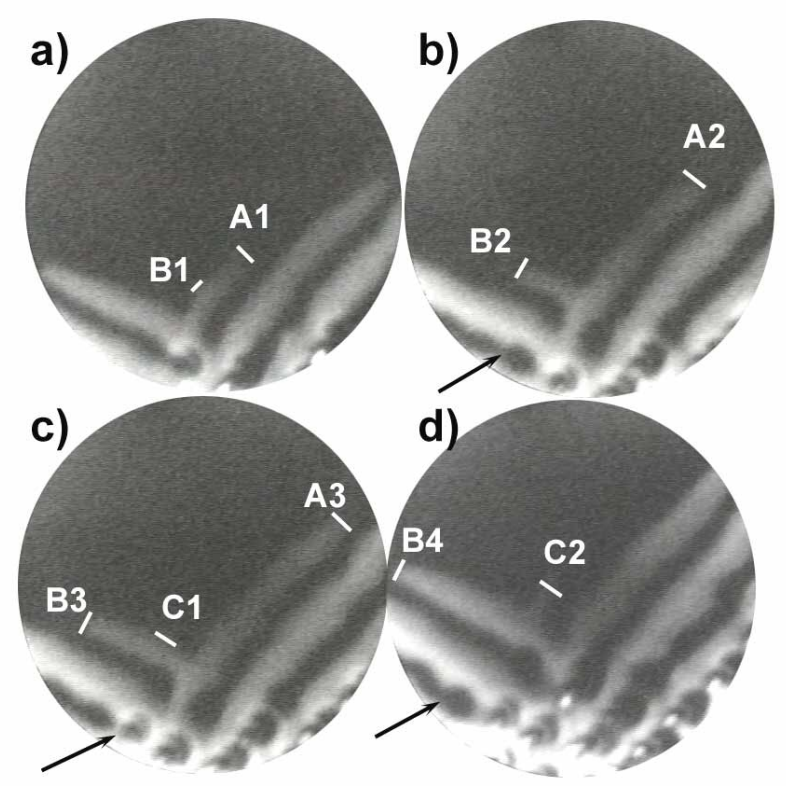

Figure 3. Real time LEEM images taken of a SOI template during decomposition at $980^{\circ} \mathrm{C}$. The time interval between successive images is 5 seconds. In this imaging mode, silicon regions appear dark while the exposed oxide is light. Labels A, B, C indicate the endpoints of trenches in the template layer. The black arrows indicate capillary instabilities in the silicon ridge which cause it to break up into individual silicon nanocrystals.

As the surface evolves from figure $3 \mathrm{a}$ to $3 \mathrm{~b}$, one can observe a trench extending from position A1 to A2 along the [130] direction; at the same time another trench forms at B1 and grows to B2 along the [310] direction. This process proceeds, in figures $3 \mathrm{c}$ and $3 \mathrm{~d}$, to form a network of trenches in $<130>$ directions. As two adjacent trenches expand in width, the $\mathrm{Si}$ atoms leaving the trench area collect on the Si ridge between them. Finally, the Rayleigh instability [21] in the ridges causes them to break into individual Si nanocrystals, as indicated by the black arrows in figures $3 b-d$. These Si islands must line up along the $<130>$ directions in which the ridges were originally oriented.

It has been previously observed that nucleation of agglomerated patterns in SOI occurs at thin spots in the template layer or pinhole defects [10]. Any deviation from a (100) surface lowers the kinetic barrier to the formation of a (311) facet. Therefore, by designing the locations of the nucleation sites, e.g., lithographically defining a pattern, the Si nanocrystals can be forced to align [14]. An example of this kind of forced ordering is shown in figure 4. SOI was patterned with template strips aligned in the $<100>$ direction and then heated to dewet the silicon. Silicon islands form in a line along the $<100>$ strip. This capability offers great potential flexibility in the design of specific device architectures for nano-electronic systems either through use as a template, as for the ordering of nanotubes discussed in the next section, or for the precise ordering and positioning of individual Si nanocrystals.

For decompositions performed at elevated temperatures $\left(\sim 1200^{\circ} \mathrm{C}\right)$, no ordering of Si islands along preferred directions is observed and $\mathrm{Si}$ (311) facets are no longer preferred. Islands are randomly distributed around the starting defect sites. We believe that the surface energy anisotropy is reduced by the increasing entropy contribution at higher temperatures. 


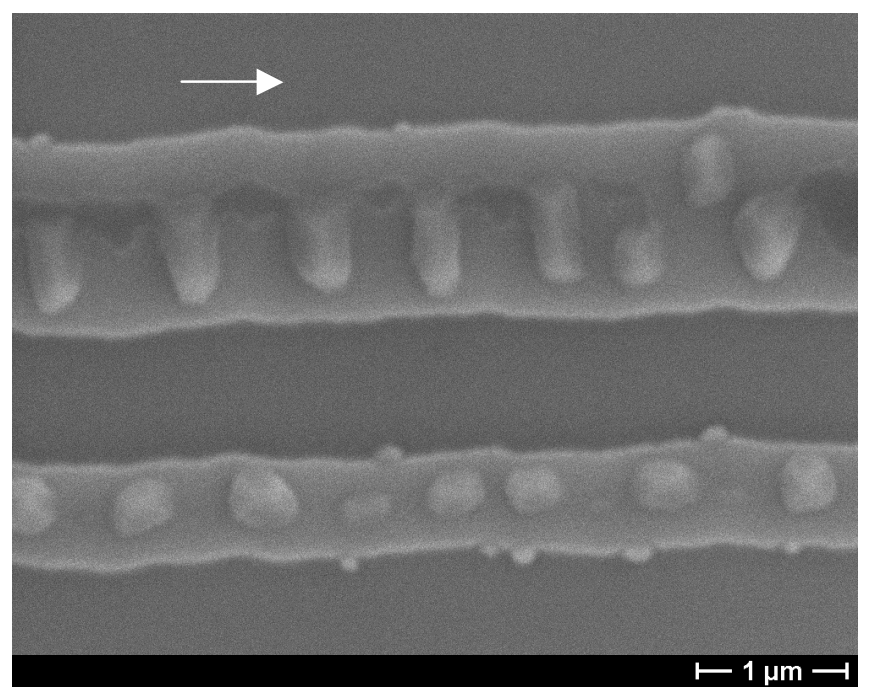

Figure 4. SEM image of a line of silicon islands formed by decomposition along a $<100>$ direction.

Furthermore, depositing several monolayers of Ge on thin SOI decreases the decomposition temperature and makes the decomposition more isotropic. We know that Ge weakens the bonds and roughens the surface at lower temperature [22, 23]. It is thus likely that the surface free energy of Ge-covered Si becomes more isotropic than that of pure Si.

\section{$\underline{\text { Self-assembly of nanotube networks on agglomerated SOI }}$}

While fundamentally interesting, carbon nanotubes have yet to reach their potential for practical electronic applications. This is largely due to the difficulty with which they are ordered or aligned. The most successful results in directed growth of nanotubes have made carbon nanotube networks grown from lithographically defined features [24-26] [27]. Because the main attribute required for these substrates is that they have asperities for the nanotubes to attach, the use of a self-ordering template such as the decomposed SOI eliminates a costly lithography step. As demonstrated in the previous section, however, the substrate can be patterned to align the growth of the Si nanocrystals to arrange the nanotube network into any desired pattern.

In these previous studies, catalyst is selectively positioned on the top of pillars such that nanotubes grow between pillars [24] [27]. When catalyst is distributed evenly over the sample, nanotubes grow both on top of the pillars and in the areas between the pillars [26]. In our experiment, we deposit catalyst over the entire surface of the Si nanocrystals and on the oxide between the nanocrystals. Nanotubes can in principle grow anywhere and start from any location, at the top of the islands or otherwise. Nanotubes appear, in fact, to energetically favor growth on the oxide [27]. Yet we observe a strong preference for nanotubes to form between the tops of the $\mathrm{Si}$ islands as seen in Figure 5.

The Si nanocrystals are about $90 \mathrm{~nm}$ high and 100-150nm wide, with $200 \mathrm{~nm}$ base-to-base spacing. With this geometry, the nanocrystals will likely interfere with the flow of the carbon feed gas. At our growth conditions, the mean free path of the gas molecules is on the same order as the separation of the $\mathrm{Si}$ islands, and the feed gas may not be able to reach the bottom areas 

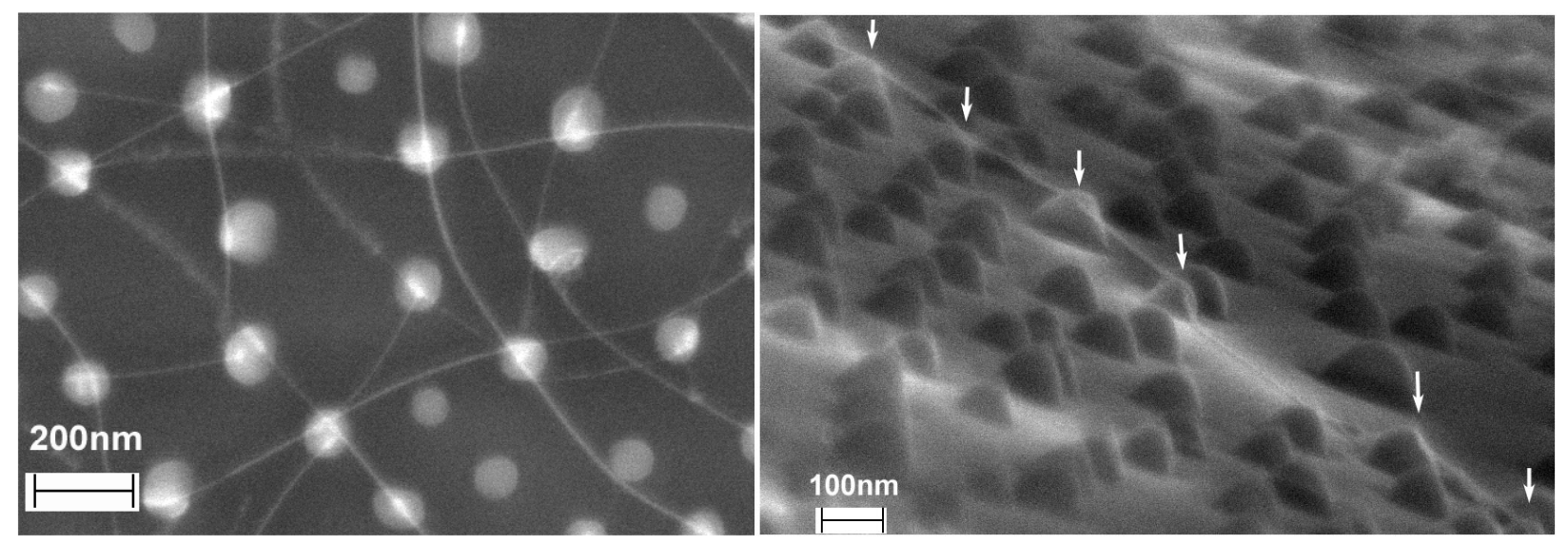

Figure 5. SEM images of carbon nanotubes deposited on an array of silicon nanocrystals formed by SOI decomposition: a) Top view shows the tubes form a network that joins the Si islands; b) Grazing angle view shows that the nanotubes connect the islands at their apexes. A transition from peak-to-peak growth to surface growth can be seen between the third and fifth arrows, counting from left to right.

between the Si nanocrystals, creating a stagnant zone. To confirm this model, we examine the effect of the Si nanocrystals on the flow pattern for our CVD growth conditions, using computational fluid dynamics (CFD) modeling software (Fluent).

In our CVD growth, the methane flow is laminar [28]. Because the growth is performed at near-atmospheric pressures, the gas transport is dominated by molecule-molecule collisions, and the flow can be described by continuum theory. As a simple first approximation, we consider only geometric effects in the simulation.

The simulation is done in two dimensions $(\mathrm{x}, \mathrm{z})$ with an array of rectangular islands on the sample surface. The islands are $90 \mathrm{~nm}$ high, $120 \mathrm{~nm}$ wide, with $200 \mathrm{~nm}$ spacing on the left side of the model substrate and $400 \mathrm{~nm}$ spacing on the right. An illustration of this arrangement is shown in figure 6 . The gas flow far away from the substrate has a uniform velocity of $3.3 \mathrm{~mm} / \mathrm{s}$, as estimated from our growth parameters [28]. Figure 6 shows that the islands introduce vertical

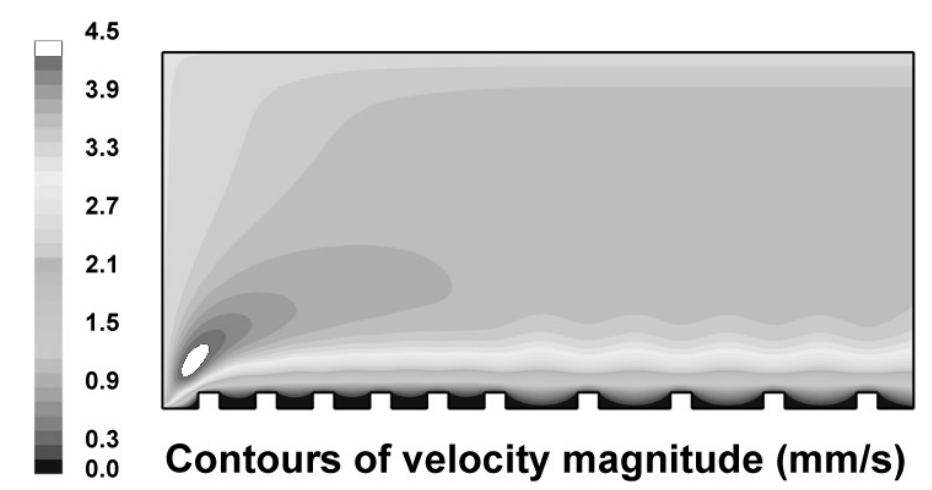

Figure 6. CFD simulation of methane flow patterns over a decomposed SOI substrate with two different nanocrystal densities. The model islands are $90 \mathrm{~nm}$ high and $120 \mathrm{~nm}$ wide. On the right half of the substrate, the islands are separated by $400 \mathrm{~nm}$. On the left half, the islands are more densely packed (200 $\mathrm{nm}$ spacing. 
oscillations in the flow pattern. An individual protrusion leaves a wake in the gas flow. The flow will regain its laminar character after a characteristic distance which is a function of the flow parameters. If the wake has not recovered before encountering the next obstacle, a stagnant region exists between the two protrusions, as seen in earlier studies of flow on rough surfaces [29]. Figure 6 shows a plot of the flow field resulting from our model.

Our simulation reveals stagnant regions (dark colored) between the islands suggesting that nanotube growth may be inhibited by the lack of methane feed gas. Although there are catalyst particles on the oxide between the islands, they are not able to capture the carbon feedstock. If the island spacing is increased, the gas flux increases near the oxide surface, as shown for the right half of the model. Chances for nanotubes to start growing from catalyst particles on the oxide should be larger as the island spacing increases. An example of this is seen in figure 2a, where nanotubes extend between the island tops for closely spaced islands yet, as soon as a large gap appears, the nanotube grows on the oxide surface.

\section{CONCLUSIONS}

In conclusion, the template layer of ultra-thin SOI is found to dewet the oxide and agglomerate into an ordered array of Si nanocrystals at elevated temperatures in ultrahigh vacuum. We have used real-time LEEM imaging combined with first-principles calculations to identify and quantify the mechanism of nanocrystal formation and self-organization during this process. The self-organization is driven by a large surface energy anisotropy, which leads to predominant formation of nanocrystals bounded by (311) facets, as confirmed by first-principles calculations. In addition, we show that carbon nanotubes can be made to grow preferentially on the apexes of these structures by manipulating the island spacing. Lithographic patterning of SOI template layers opens the possibility for designing nanocrystal arrays as templates for selfassembling nanotube network growth.

\section{ACKNOWLEDGEMENTS}

Aspects of the research reviewed here that were performed at UW-Madison were supported in part by DOE and in part by NSF. Feng Liu's work is supported by DOE.

\section{REFERENCES}

1. W.M. Kane, J.P. Spratt, and L.W. Hershinger, J. Appl. Phys. 37, 2085-2089 (1966).

2. P. Scharnhorst, Surf. Sci. 15, 380-6 (1969).

3. R.E. Hummel, R.T. DeHoff, S. Matts-Goho, and W.M. Goho, Thin Solid Films 78, 1-14 (1981).

4. J.-Y. Kwon, T.-S. Yoon, K.-B. Kim, and S.-H. Min, J. Appl. Phys. 93, 3270-8 (2003).

5. D.J. Srolovitz, W. Yang, and M.G. Goldiner in Polycrystalline Thin Films: Structure, Texture, Properties, and Applications II, edited by H.J. Frost, M.A. Parker, C.A. Ross, and E.A. Holm, (Mater. Res. Soc. Symp. Proc. 403, Pittsburgh, Pa, 1996), pp. 3-13.

6. A.R. Woll, P. Moran, E.M. Rehder, B. Yang, T.F. Kuech, and M.G. Lagally in Current Issues in Heteroepitaxial Growth - Stress Relaxation and Self Assembly, edited by E. Stach, E. Chason, R. Hull, and S. Bader, (Mater. Res. Soc. Symp. Proc. 696, Pittsburgh, Pa, 2002), pp. 119-24. 
7. S. Yamamoto, S. Masuda, H. Yasufuku, N. Ueno, Y. Harada, T. Ichinokawa, M. Kato, and Y. Sakai, J. Appl. Phys. 82, 2954-2960 (1997).

8. Y. Ding, S. Yamamuro, D. Farrell, and S.A. Majetich, J. Appl. Phys. 93, 7411-7413 (2003).

9. $\quad$ K. Yamagata and T. Yonehara, Appl. Phys. Lett. 61, 2557-9 (1992).

10. Y. Ono, M. Nagase, M. Tabe, and Y. Takahashi, Jpn. J. Appl. Phys, Pt 1 34, 1728-35 (1995).

11. N. Sugiyama, T. Tezuka, and A. Kurobe, J. Cryst. Growth 192, 395-401 (1998).

12. B. Legrand, V. Agache, T. Melin, J.P. Nys, V. Senez, and D. Stievenard, J. Appl. Phys. 91, 106-11 (2002).

13. R. Nuryadi, Y. Ishikawa, and M. Tabe, Appl. Surf. Sci. 159-160, $121-6$ (2000).

14. Y. Ishikawa, Y. Imai, H. Ikeda, and M. Tabe, Appl. Phys. Lett. 83, 3162-4 (2003).

15. D.J. Eaglesham, A.E. White, L.C. Feldman, N. Moriya, and D.C. Jacobson, Phys. Rev. Lett. 70, 1643-6 (1993).

16. J.M. Bermond, J.J. Metois, X. Egea, and F. Floret, Suf. Sci. 330, 48-60 (1995).

17. K.D. Brommer, M. Needels, B.E. Larson, and J.D. Joannopoulos, Phys. Rev. Lett. 68, 1355-8 (1992).

18. J. Dabrowski, H.-J. Mussig, and G. Wolff, Phys. Rev. Lett. 73, 1660-3 (1994).

19. A. Laracuente, S.C. Erwin, and L.J. Whitman, Phys. Rev. Lett. 81, 5177-80 (1998).

20. B. Yang, P. Zhang, M.G. Lagally,G.-H. Lu, M. Huang, and F. Liu, Submitted to Physical Review Letters.

21. L. Rayleigh, Proc. London Math. Soc. 10, 4 (1878).

22. F. Liu, F. Wu, and M.G. Lagally, Chem. Rev. 97, 1045-1061 (1997).

23. F. Wu and M.G. Lagally, Phys. Rev. Lett., 75, 2534 (1995).

24. N.R. Franklin and H. Dai, Adv. Mat. 12, 890-894 (2000).

25. T. Kitajima, B. Liu, and S.R. Leone, Appl. Phys. Lett. 80, 497 (2002).

26. Y. Homma, Y. Kobayashi, T. Ogino, and T. Yamashita, Appl. Phys. Lett. 81, 2261 (2002).

27. Y.J. Jung, Y. Homma, T. Ogino, Y. Kobayashi, D. Takagi, B. Wei, R. Vajtai, and P.M. Ajayan, J. Phys. Chem. B 107, 6859-6864 (2003).

28. At atmospheric pressure, the flow velocity $v$ of the methane gas is calculated to be 0.33 $\mathrm{cm} / \mathrm{s}$ (the methane mass flow rate is $400 \mathrm{sccm}$ and the diameter $d$ of the furnace tube is 2 inches). We estimate that the Reynolds number (Re) of methane flow in the furnace tube is smaller than 10 using the formula $\operatorname{Re}=\rho v d / \eta$, where $\rho$ is the density of methane gas $(\rho$ $=178 \mathrm{~g} / \mathrm{m}^{3}$ at $900^{\circ} \mathrm{C}$ and $\left.1 \mathrm{~atm}\right)$, and $\eta$ is the gas viscosity $\left(\eta=1.8 \times 10^{-5} \mathrm{~kg} / \mathrm{m}-\mathrm{s}\right)$. Also, the mean free path, $\lambda$, of methane molecules is very small compared to the tube diameter during growth $\left(\lambda=5 \times 10^{-3} \mathrm{~cm}\right.$ for $\mathrm{N}_{2}$ at room temperature and 1 Torr $)$. The Knudsen number is much smaller than 0.01 . Thus, the gas flow can be modeled by continuum theory.

29. Y. Hu, C. Werner, and D. Li, J. Fluids Eng. 125, 871-879 (2003). 